\title{
Some Results on the Sandor-Smarandache Function
}

\author{
S. M. S. Islam ${ }^{1 *}$, A. A. K. Majumdar ${ }^{2}$ \\ ${ }^{1}$ Department of Mathematics, Hajee Mohammad Danesh Science and Technology University, \\ Dinajpur-5200, Bangladesh \\ ${ }^{2}$ Ritsumeikan Asia-Pacific University, Beppu-shi 874-8577, Japan
}

Received 21 June 2020, accepted in final revised form 7 October 2020

\begin{abstract}
Sandor introduced a new Smarandache-type function, denoted by $\operatorname{SS}(n)$, and is called the Sandor-Smarandache function. When $n$ is an odd (positive) integer, then $\operatorname{SS}(n)$ has a very simple form, as has been derived by Sandor himself. However, when $n$ is even, then the form of SS $(n)$ is not simple, and remains an open problem. This paper finds $\operatorname{SS}(n)$ for some special cases of $n$. Particular attention is given to values of the general forms $S S(2 m p)$, $S S(6 \mathrm{mp}), S S(60 \mathrm{mp})$ and $S S(420 \mathrm{mp})$, where $m$ is any (positive) integer and $p$ is an odd prime. Some particular cases have been treated in detail. In Section 4, some remarks are observed.
\end{abstract}

Keywords: Arithmetic function; Binomial coefficient, Diophantine equation, SandorSmarandache function

(C) 2021 JSR Publications. ISSN: 2070-0237 (Print); 2070-0245 (Online). All rights reserved. doi: http://dx.doi.org/10.3329/jsr.v13i1.47721 J. Sci. Res. 13 (1), 73-84 (2021)

\section{Introduction}

F. Smarandache is a Romanian-American mathematician, who, in late nineteen seventies, raised some interesting problems from a wide and diverse field of mathematics, and are commonly known as the Smarandache notions. Notable among them is the Smarandache function, which has been studied extensively by the mathematicians and number theorists throughout the world. The characteristic of the function is that it is not a traditional arithmetic function of number theory. Since then more arithmetic functions of Smarandache-type appeared in literature. Possibly, the latest Smarandache-type arithmetic function is the Sandor-Smarandache function, posed by Sandor [1] in 2001, and would be denoted by $S S(n)$. For a long time, the function remained in oblivion. As far as we know, the first systematic study of the function was made by Majumdar [2].

The Sandor-Smarandache function, $S S(n)$, is defined as follows:

$S S(n)=\max \left\{k: 1 \leq k \leq n-2, n\right.$ divides $\left.\left(\begin{array}{l}n \\ k\end{array}\right)\right\}, n \geq 3$,

*Corresponding author: $\underline{\text { sislam.math@gmail.com }}$ 
where $C(n, k) \equiv\left(\begin{array}{l}n \\ k\end{array}\right)=\frac{n !}{k !(n-k) !}$ are the binomial coefficients, and by convention, $S S(1)=1, S S(2)=1, S S(3)=1, S S(4)=1, S S(6)=1$.

The following result is due to Sandor [1].

Lemma 1.1: If $n(\geq 3)$ is an odd integer, then $S S(n)=n-2$.

Corollary 1.1: $S S(p)=p-2$ for any prime $p \geq 3$.

Proof: is trivial, and is left as an exercise for the reader.

Corollary 1.2: For any two odd integers $m$ and $n, S S(m n)=m n-2$.

Corollary 1.3: For any odd integer $n, S S\left(n^{m}\right)=n^{m}-2$ for any integer $m \geq 1$.

Sandor [1] leaves the case when $n$ is even as a "difficult problem". This paper considers this case in Section 3, where $S S(n)$ for some particular cases is derived. In Section 2, some background material is given that would be needed later. The main results of the paper are given in Section 3, where the explicit expressions of $S S(2 m p), S S(6 m p)$, $S S(60 \mathrm{mp})$ and $S S(420 \mathrm{mp})$ are derived, where $m$ is a positive integer and $p$ is an odd prime. Some remarks are mentioned in the Section 4. Some concluding remarks are made in final Section 5 .

\section{Background Material}

In finding the expressions of $S S(n)$ for different forms of $n$ in the next section, we would frequently encounter the Diophantine equation of the form $a x+b y=c$. In this context, Lemma 2.1 is relevant.

Lemma 2.1: The Diophantine equation $a x+b y=c$ has an (integer) solution if and only if $c$ is divisible by $d \equiv(a, b)$. Moreover, if $\left(x_{0}, y_{0}\right)$ is a solution, then there are infinite number of solutions, given parametrically by $x=x_{0}+\left(\frac{b}{d}\right) t, y=y_{0}+\left(\frac{-a}{d}\right) t$ for any integer $t$.

Proof: See, for example, Gioia [3].

\section{Main Results}

The following results are found.

Lemma 3.1: Let $n=2 m$, where $m(\geq 4)$ is an integer, not divisible by 3 . Then, $S S(n)=n-3$.

Proof: Consider the expression

$C(n, n-3) \equiv \frac{2 m(2 m-1)(2 m-2)}{2 \times 3}=\frac{2 m(2 m-1)(m-1)}{3}$.

Here, since $m$ is not divisible by 3 , one of $m-1$ and $2 m-1$ must be divisible by 3 .

Corollary 3.1: For any even integer $n$, not divisible by $3, S S\left(n^{m}\right)=n^{m}-3$ for any integer $m \geq 1$.

Proof: follows readily from Lemma 3.1.

Lemma 3.2: For any prime $p \geq 5, S S(2 m p)=2 m p-3$ for any integer $m \neq 3$.

Proof: Considering the expression

$C(2 m p, 2 m p-3) \equiv \frac{2 m p(2 m p-1)(2 m p-2)}{2 \times 3}=\frac{2 m p(2 m p-1)(m p-1)}{3}$, 
the result follows, since one of $2 m p-1$ and $m p-1$ is divisible by 3 .

Note that, Lemma 3.2 above holds when $m=2, p=2$.

The case when $n$ is a multiple of 3 is considered; more precisely, we consider the case of the function of the form $S S(6 m)$, where $m \geq 1$ is an integer. From the expression $6 m \frac{(6 m-1)(6 m-2)}{3 !}=6 m \frac{(6 m-1)(3 m-1)}{3}$,

it is clear that $S S(6 m) \leq 6 m-4$. We now prove the following result.

Lemma 3.3: Let $n=6 m, m \geq 1$. Then, for any integer $s \geq 0$,

(1) $S S(n)=n-4$, if $m=4 s+3$,

(2) $S S(n)=n-5$, if $m$ is any one of the four forms (for some integers $\alpha \geq 0, \beta \geq 1, \gamma \geq 0$, $\mu \geq 2)$ :

$m=2(5 \alpha+4) ; m=10 \beta+3, \beta \neq 4 s ; m=2(5 \gamma+3) ; m=10 \mu+1, \mu \neq 2 s+1$.

Proof: To prove part (1), consider the simplified expression below.

$C(6 m, 6 m-4) \equiv 6 m\left[\frac{(6 m-1)(3 m-1)(2 m-1)}{4}\right]$.

Now, in order that the numerator of the term inside the square bracket is divisible by 4 , $3 m-1$ must be divisible by 4 , that is, the Diophantine equation

$3 m=4 a+1$ for some integer $a(\geq 1)$,

whose solution is $m=4 s+3, s \geq 0$ is to be solved. Thus, if $m=4 s+3$, then $S S(n)=n-4$. To prove part (2), let $m \neq 4 s+3$, and the expression which is reduced as follows:

$C(6 m, 6 m-5) \equiv 6 m\left[\frac{(6 m-1)(3 m-1)(2 m-1)(3 m-2)}{2 \times 5}\right]$.

Here, $6 m-1$ and $2 m-1$ are odd, and only one of the two numbers $3 m-1$ and $3 m-2$ is even. Moreover, $m$ should be such that, one of $6 m-1$ and $2 m-1$ is divisible by 5 . Thus, there are four possibilities, and consequently, four sets of equations, namely,

(1) $2 m=5 a+1$ and $3 m=2 b+2$ (for some integers $a \geq 1$ and $b \geq 1$ ),

(2) $2 m=5 a+1$ and $3 m=2 b+1$ (for some integers $a \geq 1$ and $b \geq 1$ ),

(3) $6 m=5 a+1$ and $3 m=2 b+2$ (for some integers $a \geq 1$ and $b \geq 1$ ),

(4) $6 m=5 a+1$ and $3 m=2 b+1$ (for some integers $a \geq 1$ and $b \geq 1$ ),

are to be solved with respective solutions

$m=2(5 \alpha+4) ; m=10 \beta+3, \beta \neq 4 s ; m=2(5 \gamma+3) ; m=10 \mu+1$,

where $\alpha \geq 0, \beta \geq 1, \gamma \geq 0, \mu \geq 2$ are integers with $\mu \neq 2 s+1$.

For example, part (1) of Lemma 3.3 for $m=3$ shows that 18 divides $18\left(\frac{17 \times 16 \times 15}{2 \times 3 \times 4}\right)$.

Thus, $S S(18)=14$. Similarly, $S S(42)=38$. Part (2) of Lemma 3.3 provides the following values:

$S S(48)=43, S S(108)=103, S S(168)=163, S S(228)=223, S S(78)=73, S S(198)=193$,

$S S(36)=31, S S(96)=91, S S(156)=151, S S(216)=211, S S(126)=121, S S(246)=241$.

Note that, part (2) of Lemma 3.3 applies when $m \neq 4 s+3$.

The two lemmas below give expressions of $S S(6 \mathrm{mp})$ under certain conditions.

Lemma 3.4: Let $p$ be a prime and $m(\geq 1)$ be an integer. Let $p$ and $m$ be such that one of them is of the form $4 a+1$, and the other is of the form $4 b+3(a \geq 1, b \geq 1)$. Then,

$S S(6 m p)=6 m p-4$.

Proof: Consider the expression 
$C(6 m p, 6 m p-4) \equiv 6 m p\left[\frac{(6 m p-1)(3 m p-1)(2 m p-1)}{4}\right]$.

Now, to prove the lemma, we have to find the condition that 4 divides $3 m p-1$, that is

$3 m p-1=4 s$ for some integer $s \geq 1$.

Now, if $m=4 a+1$ (for some integer $a \geq 1$ ), then

$3 m p-1=3(4 a+1) p-1=12 a p+(3 p-1)$,

which is divisible by 4 if 4 divides $3 p-1$, giving rise to the Diophantine equation

$3 p-1=4 x$ for some integer $x \geq 1$.

The solution of the above equation is $p=4 b+3, b \geq 1$ being an integer.

Next, let $m=4 b+3$ (for some integer $b \geq 1$ ); then

$3 m p-1=3(4 b+3) p-1=12 b p+(9 p-1)$,

which is divisible by 4 if 4 divides $9 p-1$. Thus, a Diophantine equation is formed as follows:

$9 p-1=4 y$ for some integer $y \geq 1$.

The solution of the above equation is $p=4 a+1, a \geq 1$ being an integer.

All these complete the proof of the lemma.

To illustrate Lemma 3.4, let $m=5$ (which is of the form $4 a+1$ ) and $p=3$ (which is of the form $4 b+3)$. Then, $S S(90)=86$. Similarly, with $m=5$ and $p=7$, it is found that $S S(210)=206$. Again, with $m=3$ and $p=13$, we get $S S(234)=230$. And with $m=7$ and $p=13$, we get $S S(546)=542$. The example shows that Lemma 3.4 holds true even when $a=0$ and $b=0$.

Lemma 3.5: Let $p(\geq 3)$ be a prime with $p \neq 5$, and $m(\geq 1)$ be an integer such that the condition of Lemma 3.4 is not satisfied, and $m$ is not a multiple of 5. Then,

$S S(6 m p)=6 m p-5$.

Proof: We start with the simplified form

$C(6 m p, 6 m p-5) \equiv 6 m p\left[\frac{(6 m p-1)(3 m p-1)(2 m p-1)(3 m p-2)}{2 \times 5}\right]$.

Now, one of $3 m p-1$ and $3 m p-2$ is even, and one of the four factors, namely, $6 m p-1$, $3 m p-1,2 m p-1$ and $3 m p-2$, is divisible by 5 (since $m p$ is not divisible by 5 ).

Some consequences of Lemmas 3.2 -3.5 are given below.

Corollary 3.2: Let $p \neq 5$ be a prime. Then,

$S S(6 p)= \begin{cases}6 p-4, & \text { if } p=4 s+3, s \geq 0 \\ 6 p-5, & \text { if } p=4 s+1, s \geq 3\end{cases}$

Proof: The first part follows from Lemma 3.4. Now, with $m=1$, the condition in Lemma 3.5 is satisfied if and only if $p=4 s+1$. Thus, the second part also follows.

Corollary 3.3: $S S(12 p)=12 p-5$ for any prime $p \neq 5$.

Proof: follows readily from Lemma 3.5.

Corollary 3.4: Let $p \neq 5$ be a prime. Then,

$S S(18 p)= \begin{cases}6 p-4, & \text { if } p=4 s+1, s \geq 0 \\ 6 p-5, & \text { otherwise }\end{cases}$

Proof: The proof follows from Lemma 3.4, and Lemma 3.5.

Corollary 3.5: $S S(24 p)=24 p-5$ for any prime $p \neq 5$. 
Proof: follows immediately from Lemma 3.5.

Now, the case when $m=5$, that is, the case of $S S(30 p)$ is considered. It can easily be verified that $S S(30 p) \neq 30 p-3$. But the following result is found.

Corollary 3.6: $S S(30 p)=30 p-4$, if the prime $p$ is of the form $p=4 s+3, s \geq 0$.

Proof: follows from Lemma 3.4.

A few examples are given to demonstrate the results of Corollary 3.2-Corollary 3.6:

$S S(42)=38, S S(78)=73$ (Corollary 3.2), SS $(36)=31, S S(84)=79$ (Corollary 3.3), $S S(198)=193$ (Corollary 3.4), SS(72) $=67, S S(168)=163$ (Corollary 3.5), and from Corollary 3.6, $S S(90)=86, S S(330)=326$. Note that Corollary 3.6 is valid for $s=0$.

Lemma 3.6: $S S(30 p) \neq 30 p-5$ for any prime $p$.

Proof: Consider the expression

$C(30 p, 30 p-5) \equiv 30 p\left[\frac{(30 p-1)(15 p-1)(10 p-1)(15 p-2)}{2 \times 5}\right]$.

Noting that none of the four factors in the numerator inside the square bracket is divisible by 5 , the result follows.

Lemma 3.7: $S S(30 p) \neq 30 p-6$ for any prime $p$.

Proof: From the expression

$C(30 p, 30 p-6) \equiv 30 p\left[\frac{(30 p-1)(15 p-1)(10 p-1)(15 p-2)(6 p-1)}{4 \times 3}\right]$,

the result follows, since neither $15 p-2$ nor $6 p-1$ is divisible by 3 .

Lemma 3.8: $S S(30 p)=30 p-7$, if the prime $p$ is of the form $p=4 s+1, s \geq 1$.

Proof: Consider the expression

$C(30 p, 30 p-7)=30 p\left[\frac{(30 p-1)(15 p-1)(10 p-1)(15 p-2)(6 p-1)(5 p-1)}{2 \times 7}\right]$.

Now, one of $15 p-1$ and $15 p-2$ is even. Thus, the term inside the square bracket on the right is an integer.

Now, the attention is confined to the function $S S(60 p)$, where $p$ is a prime.

Lemma 3.9: for any prime $p$, (1) $S S(60 p) \neq 60 p-4$, (2) $S S(60 p) \neq 60 p-5$.

Proof: Part (1) follows from Lemma 3.4. The proof of part (2) is similar to that of Lemma 3.6, and is omitted here.

Lemma 3.10: $S S(60 p)=60 p-6$, if the prime $p$ is of the form $p=3 s+2, s \geq 1$.

Proof: Consider

$C(60 p, 60 p-6)=60 p\left[\frac{(60 p-1)(30 p-1)(20 p-1)(15 p-1)(12 p-1)}{6}\right]$.

Now, when $p$ is an odd prime, then $15 p-1$ is even. It is thus sufficient to find the condition that 3 divides $20 p-1$, that is, to solve the Diophantine equation

$20 p=3 a+1$ for some integer $a \geq 1$.

The solution of the above equation is $p=3 s+2, s \geq 1$, which is the desired condition.

Lemma 3.11: Let $p(\geq 11)$ be a prime such that $p \neq 3 s+2$ for any $s \geq 1$. Then, $S S(60 p)=60 p-7$.

Proof: Simplfy $C(60 p, 60 p-7)$ as follows :

$C(60 p, 60 p-7) \equiv 60 p\left[\frac{(60 p-1)(30 p-1)(20 p-1)(15 p-1)(12 p-1)(10 p-1)}{7}\right]$.

The result is now evident from the above expression (since 7 does not divide $p$ ). 
The following examples illustrate the results of Lemma 3.8, Lemma 3.10 and Lemma 3.11:

$S S(150)=143, S S(390)=383, S S(510)=503, S S(870)=863$.

$S S(300)=294, S S(780)=773, S S(1140)=1133$.

Next, we consider the case of $S S(60 \mathrm{mp})$. The following two lemmas are easy to prove, and are stated without proof.

Lemma 3.12: For any integer $m(\geq 1)$ and for any prime $p(\geq 3), S S(60 m p) \neq 60 m p-4$.

Lemma 3.13: For any integer $m(\geq 1)$ and for any prime $p(\geq 3), S S(60 \mathrm{mp}) \neq 60 \mathrm{mp}-5$.

However, $S S(60 m p)=60 m p-6$, which is proved below.

Lemma 3.14: Let $m(\geq 1)$ be an integer and $p(\geq 5)$ be a prime such that

$m p=6 s+5$ for some integer $s \geq 1$.

Then,

$S S(60 m p)=60 m p-6$.

Proof: We consider the expression

$C(60 m p, 60 m p-6) \equiv 60 m p\left[\frac{(60 m p-1)(30 m p-1)(20 m p-1)(15 m p-1)(12 m p-1)}{6}\right]$.

Then, 3 must divide $20 m p-1$ and 2 must divide $15 m p-1$. That is,

$20 m p=3 x+1,15 m p=2 y+1$ for some integers $x(\geq 1)$ and $y(\geq 1)$.

From the two above Diophantine equations, we get the following one:

$9 x=8 y+1$,

with the solution

$x=8 a+1, y=9 b+1$ for any integers $a(\geq 1)$ and $b(\geq 1)$.

Therefore,

$20 m p=3(8 a+1)+1=4(6 a+1)$,

that is,

$5 m p=6 a+1, a \geq 1$.

The solution of the above equation is $m p=6 s+5$ ( $s \geq 0$ being an integer).

The proof of Lemma 3.14 shows that, $S S(60 \mathrm{mp})=60 \mathrm{mp}-6$ (for some integer $m \geq 1$ and prime $p \geq 5$ ) if and only if $m p$ satisfies the relationship (3.1) holds. From (3.1), we see that it has a solution only if $m$ is an odd integer; moreover, for any odd integer $m$ fixed, (3.1) has always a solution, which can then be solved to find $p$. For example, when $m=1$, the condition of Lemma 3.14 becomes

$p=6 s+5$ for some integer $s \geq 0$.

When $s=0, p=5$, and hence, by Lemma 3.14, $S S(60 \times 1 \times 5)=244$. The next solution is $p=17$, with $S S(60 \times 1 \times 17)=S S(1020)=1014$. Other solutions are $p=23$ with $S S(1380)=1374, p=29$ with $S S(1740)=1734, p=41$ with $S S(2460)=2454$, and $p=43$ with $S S(2820)=2814$. By Lemma 2.1, there is no solution of (3.1) when $m=2,3,4$. However, with $m=5$, the condition reads as

$5 p=6 s+5$,

which has a solution, namely, $p=6 t+7$ ( $t \geq 0$ being an integer). Now, the first solution of the equation $p=6 t+7$ is $p=7$ with $S S(60 \times 5 \times 7)=S S(2100)=2094$. We find that the next solution is $p=13$ with $S S(3900)=3894$. With $m=7$, the condition becomes $7 p=6 s+5$, 
with the solution $p=6 v+5$ ( $v \geq 0$ being an integer). The first few primes in this case are (excluding the repeating $p=5$ ), $p=11,17,23,29$, with $S S(4620)=4614, S S(7140)=7134, S S(9660)=9654, S S(12180)=12174$.

And when neither $m$ nor $p$ is a multiple of 7 , and $m p \neq 6 s+5$ for any integer $s \geq 1$, then $S S(60 \mathrm{mp})=60 \mathrm{mp}-7$.

For example, with $m=1$, the least admissible value of $p$ is $p=13$, which gives the value $S S(780)=773$. Again, with $m=1$ and $p=19$, we get $S S(1140)=1133$; and $m=1$ and $p=31$ provides the function $S S(1860))=1853$. Similarly, $m=3$ and $p=13$ give $S S(2340)$ $=2333 ; m=3$ and $p=19$ suggest $S S(3420)=3413$; and $m=3$ and $p=31$ give the function $S S(55800)=5573$.

Attention is now focussed to the function SS(420mp).

The three lemmas are easy to prove, and proofs are left for the reader.

Lemma 3.15: $S S(420 m p) \neq 420 m p-3$ for any integer $m(\geq 1)$ and any prime $p$.

Lemma 3.16: $S S(420 \mathrm{mp}) \neq 420 \mathrm{mp}-4$ for any integer $m(\geq 1)$ and any prime $p$.

Lemma 3.17: $S S(420 \mathrm{mp}) \neq 420 \mathrm{mp}-5$ for any integer $m(\geq 1)$ and any prime $p$.

Lemma 3.18: Let $m(\geq 1)$ be an integer and $p(\geq 5)$ be a prime such that $m p=6 s+5$ for some integer $s \geq 1$.

Then, $S S(420 m p)=420 m p-6$.

Proof: Consider the expression

$C(420 m p, 420 m p-6)=420 m p\left[\frac{(420 m p-1)(210 m p-1)(140 m p-1)(105 m p-1)(84 m p-1)}{6}\right]$.

Now, it is required that 2 divides $105 \mathrm{mp}-1$ and 3 divides $140 \mathrm{mp}-1$. Thus, $105 m p=2 x+1,140 m p=3 y+1$ for some integers $x(\geq 1)$ and $y(\geq 1)$, with respective solutions $m p=2 a+1, m p=3 b+2, a$ and $b$ being any integers.

Then, the combined Diophantine equation is $2 a=3 b+1$, with the solution $a=3 s+2, s(\geq 1)$.

Therefore, finally we get $m p=6 s+5$ ( $s \geq 0$ being an integer).

In Applying Lemma 3.18, the value of $m p$ as it is can be used. Then, the following values are found successively:

$S S(2100)=2094, S S(4620)=4614, S S(7140)=7134, S S(9660)=9656$,

$S S(12180)=12174, S S(17220)=17214, \operatorname{SS}(19740)=19734$.

Lemma 3.19: $S S(420 m p) \neq 420 m p-7$ for any integer $m(\geq 1)$ and any prime $p$.

Lemma 3.20: Let $m(\geq 1)$ be an integer and $p(\geq 5)$ be a prime such that $m p \neq 6 t+5$ for any integer $t \geq 1$.

Then, $S S(420 m p)=420 m p-8$, if $m p=8 s+1, s \geq 1$ being an integer.

Proof: $C(420 \mathrm{mp}, 420 \mathrm{mp}-8)$ may be simplified as follows : $C(420 m p, 420 m p-8)=$ 420mp $\left[\frac{(420 m p-1)(210 m p-1)(140 m p-1)(105 m p-1)(84 m p-1)(70 m p-1)(60 m p-1)}{8}\right]$. 
Now, in order that the term inside the square bracket is an integer, 8 must divide $105 m p-1$. Thus, $105 m p=8 x+1$ for some integer $x(\geq 1)$, with the solution $m p=8 s+1$ (for any integer $s \geq 1$ ), which is the desired condition. Note that, in Lemma 3.20, two conditions are satisfied: The first condition $m p \neq 6 t+5$ (for any integer $t \geq 1)$ ensures that $S S(420 \mathrm{mp}) \neq 420 \mathrm{mp}-6$. In applying the lemma, this condition must be kept in mind. Lemma 3.20 gives the following values:

$S S(10500)=10492$, $S S(1360)=13852, S S(20580)=20572$.

Observe that, in Lemma 3.20, $\mathrm{mp}$ must be odd. In applying the lemma, the value of $m p$, as it is, may be used.

Lemma 3.21: Let the integer $m$ and the prime $p(\geq 5)$ be such that $m p \neq 6 t+5, t \geq 1$. Then, $S S(420 m p)=420 m p-9$, if $m p=2(9 s+10), s \geq 1$ being an integer.

Proof: Consider the expression below:

C(420mp, 420mp-9)

$$
=420 m p\left[\frac{(420 m p-1)(210 m p-1)(140 m p-1)(105 m p-1)(84 m p-1)(70 m p-1)(60 m p-1)(105 m p-2)}{2 \times 9}\right] .
$$

Now, consider the case when 9 divides $140 m p-1$ and 2 divides $105 m p-2$. Thus,

$140 m p=9 x+1,105 m p=2 y+2$ for some integers $x(\geq 1)$ and $y(\geq 1)$.

The solutions of the above equations are

$m p=9 a+2, m p=2 b+2, a$ and $b$ being any integers.

Then, the combined Diophantine equation $9 a=2 b$ provides the solution

$a=2 s+2, s(\geq 1)$,

which, finally gives, $m p=6 s+5$ ( $s \geq 0$ being an integer).

Some of the values obtained from Lemma 3.21 are:

$S S(8400)=8391, S S(15960)=15951, S S(23520)=23511$.

Lemma 3.22: Let the integer $m$ and the prime $p(\geq 5)$ be such that $m p=6(10 s+9), s \geq 1$.

Then, $S S(420 \mathrm{mp})=420 \mathrm{mp}-10$.

Proof: After simplification, it is found that

$C(420 m p, 420 m p-10)$

$$
=420 m p\left[\frac{(420 m p-1)(210 m p-1)(140 m p-1)(105 m p-1)(84 m p-1)(70 m p-1)(60 m p-1)(105 m p-2)(140 m p-3)}{3 \times 4 \times 5}\right] .
$$

Consider the case when 3 divides $140 m p-3$, 4 divides $105 m p-2$, and 5 divides $84 m p-1$. Then, three Diophantine equations result as follows:

$140 m p=3 x+3,105 m p=4 y+2,84 m p=5 z+1$,

for some integers $x(\geq 1), y(\geq 1)$ and $z(\geq 1)$. The solutions of these equations are respectively

$m p=3 a+3, m p=4 b+2, m p=5 c+4, a, b$ and $c$ being any integers.

The solution of the first two equations is $a=4 d+1$, so that, $m p=6(2 d+1)(d \geq 1$ being an integer). This, coupled with the third equation gives $5 c=12 d+2$, whose solution is

$c=12 s+10, s(\geq 1)$,

which, finally gives $m p=6(10 s+9)(s \geq 0$ being an integer $)$.

Some of the values obtained from Lemma 3.22 are:

$S S(226800)=22670, S S(47880)=47870, S S(73080)=73070$. 
It may be mentioned here that, Lemma 3.18, Lemma 3.20, Lemma 3.21 and Lemma 3.22 may be used for the values of $m p$ specified (without actually identifying the prime). From Lemma 3.21 and Lemma 3.22, we see that $m p$ is even.

\section{Some Remarks}

As has been pointed out by Sandor [1], if $(n, k)=1$, then $n$ divides $C(n, k)$, which gives a sufficient condition. The proof of this result depends on the fact that $\left(\begin{array}{l}n \\ k\end{array}\right)=\frac{n}{k}\left(\begin{array}{l}n-1 \\ k-1\end{array}\right)$. However, the condition is not necessary. For example, though $(10,4) \neq 1,10$ divides $C(10,4)$. Also, note that, if $k$ divides $\left(\begin{array}{l}n-1 \\ k-1\end{array}\right)$ then $n$ divides $\left(\begin{array}{l}n \\ k\end{array}\right)$. The following result is interesting.

Lemma 4.1: Let the integers $n$ and $k(<n)$ be such that $n+1$ divides $\left(\begin{array}{c}n+1 \\ k\end{array}\right)$. Then, $n+1$ divides $\left(\begin{array}{l}n \\ k\end{array}\right)+\left(\begin{array}{c}n \\ k-1\end{array}\right)$

Proof: The proof relies on the property that $\left(\begin{array}{c}n+1 \\ k\end{array}\right)=\left(\begin{array}{l}n \\ k\end{array}\right)+\left(\begin{array}{c}n \\ k-1\end{array}\right)$.

For example, since 42 divides $\left(\begin{array}{l}42 \\ 19\end{array}\right)$, it follows from Lemma 4.1 that, 42 divides $\left(\begin{array}{l}41 \\ 19\end{array}\right)+\left(\begin{array}{l}41 \\ 18\end{array}\right)$. However, in this particular example, we see that $42 \times 41$ also divides $\left(\begin{array}{l}42 \\ 19\end{array}\right)=\left(\begin{array}{l}41 \\ 19\end{array}\right)+\left(\begin{array}{l}41 \\ 18\end{array}\right)$. And in fact, in this case, $43 \times 42 \times 41$ divides $\left(\begin{array}{l}42 \\ 18\end{array}\right)+\left(\begin{array}{l}41 \\ 18\end{array}\right)+\left(\begin{array}{l}41 \\ 19\end{array}\right)$. It may be mentioned here that, in this example, 41 divides each of $\left(\begin{array}{l}41 \\ 19\end{array}\right)$ and $\left(\begin{array}{l}41 \\ 18\end{array}\right)$; and $S S(42)=38$. Note that, though $n=41$ divides $\left(\begin{array}{c}n \\ 18\end{array}\right), n+1$ does not divide $\left(\begin{array}{c}n+1 \\ 18\end{array}\right)$.

Two other Smarandache type arithmetic functions are the Smarandache function, $S(n)$, and the pseudo Smarandache function, $Z(n)$, which are defined as follows:

$S(n)=\min \{m: n$ divides $m !\}$, $Z(n)=\min \left\{m: n\right.$ divides $\left.\frac{m(m+1)}{2}\right\}$.

Only a little is known about these two functions. Recently, a brief survey of $S(n)$ and $Z(n)$ has been done by Liu [4,5]. It is well-known that, $S(n)$ and $Z(n)$ are not multiplicative. Recall that, a function $f(n)$ is multiplicative if and only if $f(m n)=f(m) f(n)$ whenever $(m, n)=1$.

Let $p$ and $q$ be two distinct odd primes. Then, $S S(p q)=p q-2 \neq S S(p) S S(q)$. 
Thus, the Sandor-Smarandache function, like the Smarandache and the pseudo Smarandache functions, is not multiplicative. Since

$S(p)=p, Z(p)=p-1$,

it follows that each of the equations $S S(n)=S(n)+2$ and $S S(n)=Z(n)+1$ has an infinite number of solutions. Moreover, the equation

$S S(n)+S(n)=2 Z(n)$

has an infinite number of solutions, namely, $n=p$.

It is conjectured (Muller [6]) that the equation $S(n)=S(n+1)$ has no solution in integer $n$ (though Ashbacher [7] hopes that, someday, somebody would come up with a solution). It has been proved by Gou and $\mathrm{Li}[8]$ that, the equation $Z(n)=Z(n+1)$ has no solution. However, the result below can be proved.

Proposition 4.1: The equation $S S(n)=S S(n+1)$ has an infinite number of solutions.

Proof: Let $n$ be an integer of the form $n=6 m+1, m \geq 1$. Then, by virtue of Lemma 1.1 and Lemma 3.2,

$S S(n)=n-2=S S(n+1)$.

Lemma 4.2: The series $\sum_{n=1}^{\infty} \frac{1}{S S(n)}$ is divergent.

Proof: Consider the subsequence $\{S S(p)\}_{p \geq 3}$. Since, by Corollary 2.2, $S S(p)=p-2$, it follows that (see, for example, Hardy and Wright [9])

$\sum_{n=1}^{\infty} \frac{1}{S S(p)}=\sum_{n=1}^{\infty} \frac{1}{p-2}$

is divergent. Consequently, the lemma follows.

Murthy [10] and Khairnar et al. [11] have defined the Smarandache LCM ratio function (of the second type), denoted by $\operatorname{SL}(n, r)$ as follows:

Definition 4.1: For any integers $n$ and $r$ with $r \leq n$,

$S L(n, k)=\frac{[n, n-1, n-2, \ldots, n-k+1]}{[1,2,3, \ldots, k]}$,

where $\left[n_{l}, n_{2}, \ldots, n_{r}\right]$ is the LCM (least common multiple) of the $r$ integers $n_{l}, n_{2}, \ldots, n_{r}$, and $\left(n_{1}, n_{2}, \ldots, n_{r}\right)$ is their GCD (greatest common divisor).

It is indeed interesting to observe that the two functions, $S S(n)$, and $S L(n, k)$ are related through the following relationship.

Lemma 4.3: For any integers $n$ and $k$ with $k \leq n$, $\left(\begin{array}{l}n \\ k\end{array}\right)=S L(n, k) \frac{(n, n-1, \ldots, n-k+1)}{(1,2, \ldots, k)}$.

Proof: Noting that

$S L(n, k) \frac{(n, n-1, \ldots, n-k+1)}{(1,2, \ldots, k)}=\frac{n(n-1)(n-2) \ldots(n-k+1)}{(n, n-1, n-2, \ldots, n-k+1)} / \frac{k !}{(1,2,3, \ldots, k)}$

the result follows.

For example, from Lemma 4.3,

$\left(\begin{array}{l}n \\ 2\end{array}\right)=S L(n, 2) \frac{(n, n-1)}{(1,2)},\left(\begin{array}{l}n \\ 3\end{array}\right)=S L(n, 3) \frac{(n, n-1, n-2)}{(1,2,3)}$.

Since $(n, n-1)=1,(1,2)=1,(1,2,3)=1$, the following well-known formulas are found: 
$S L(n, 2)=\frac{n(n-1)}{2}, S L(n, 3)= \begin{cases}\frac{n(n-1)(n-2)}{6}, & \text { if } n \text { is odd } \\ \frac{n(n-1)(n-2)}{12}, & \text { if } n \text { is even }\end{cases}$

where, in the last formula, the fact that

$(n, n-1, n-2)= \begin{cases}1, & \text { if } n \text { is odd } \\ 2, & \text { if } n \text { is even }\end{cases}$

has been used. The above expressions of $\operatorname{SL}(n, 2)$ and $S L(n, 3)$ match with those given by Majumdar [12], and Majumdar and Islam [13,14]. In applying Lemma 3.1, calculation of $(n, n-1, n-2, \ldots, n-k+1)$ is needed.

\section{Conclusion}

This paper finds the values of the Sandor-Smarandache function $S S(n)$ for some particular cases, which reveals many interesting features. It seems that the value of $S S(n)$ depends on the prime factors of $n$. Comparison of Lemma 3.10 and Lemma 3.7 shows that $S S(30 p)$ and $S S(60 p)$ behave differently. In the more general case, starting from $S S(2 m p)$, it was gradually proceeded to find the values of $S S(6 \mathrm{mp}), S S(60 \mathrm{mp})$ and $S S(420 \mathrm{mp})$. It is shown that the function $S S(n)$ is not multiplicative. Solutions of the equation $S S(n)+S(n)=2 Z(n)$ are also derived. An interesting result, the apparently irrelevant functions $S S(n)$ and Smarandache LCM function $\operatorname{SL}(n, r)$ are related, has been proved. It is found that, given any integer $n$, there need not exists an integer $k$ such that $S S(n)=n-k$. For counter examples, we refer the reader to Lemma 3.6, Lemma 3.7, Lemma 3.9, Lemma 3.12, Lemma 3.13, and Lemmas 3.15 - 3.17. An open question may be posed as follows:

Question 5.1: Given any integer $k$, is there an integer $n$ such that $S S(n)=n-k$ ?

The paper is concluded with an alternative definition of the Sandor-Smarandache function:

$\operatorname{SS} 2(n)=\min \left\{k: 2 \leq k \leq n-1, n\right.$ divides $\left.\left(\begin{array}{c}n \\ n-k\end{array}\right)\right\}, n \geq 3$.

Note that, $S S 2(n)=k$ if and only if $S S(n)=n-k$.

\section{References}

1. J. Sandor, Smarandache Notions J. 12, 247 (2001).

2. A. A. K. Majumdar, Ganit 39, 15 (2019). https://doi.org/10.3329/ganit.v39i0.44161

3. A. A. Gioia, The Theory of Numbers (Dover Publications Inc., N.Y., 2001).

4. H. Liu, Scientia Magna 12, 132 (2017).

5. H. Liu, Scientia Magna 12, 145 (2017).

6. R. Muller, Unsolved Problems Related to the Smarandache Function (Phoenix Number Theory Publishing Co., 1993).

7. C. Ashbacher, An Introduction to the Smarandache Function (Erhus University Press, 1995).

8. S. Gou and J. Li, Scientia Magnetia 3, 81 (2003).

9. G. H. Hardy, and E. M. Wright, An Introduction to the Theory of Numbers, $5^{\text {th }}$ Edition (Oxford University Press, U.K., 2002).

10. A. Murthy, Smarandache Notions J. 14, 307 (2004).

11. S. M. Khairnar, A. W. Vyawahare, and J. N. Salunke, Scientia Magna 5, 29 (2009). 
12. A. A. K. Majumdar, Scientia Magna 11, 4 (2016).

13. A. A. K. Majumdar and S. M. S. Islam, HSTU J. Sci. Technol. 17, 119 (2019).

14. A. A. K. Majumdar and S. M. S. Islam, HSTU J. Sci. Technol. MS ID JST/19-02 (Pre-print). 